\title{
Deep Mutational Scanning: A Highly Parallel Method to Measure the Effects of Mutation on Protein Function
}

\author{
Lea M. Starita ${ }^{1,4}$ and Stanley Fields $1,2,3,4$ \\ ${ }^{1}$ Department of Genome Sciences, University of Washington, Seattle, Washington 98195; ${ }^{2}$ Department of \\ Medicine, University of Washington, Seattle, Washington 98195; ${ }^{3}$ Howard Hughes Medical Institute, Seattle, \\ Washington 98195
}

Deep mutational scanning is a method that makes use of next-generation sequencing technology to measure in a single experiment the activity of $10^{5}$ or more unique variants of a protein. Because of this depth of mutational coverage, this strategy provides data that can be analyzed to reveal many protein properties. Deep mutational scanning approaches are particularly amenable to being performed in Saccharomyces cerevisiae, given the extensive toolkit of reagents and technologies available for this organism.

Mutational analyses have long been critical for determining the role of individual amino acids in proteins. Single amino acid changes can affect folding, thermodynamic stability, enzymatic activity, interactions, posttranslational modifications, and other properties. Previous efforts to determine the consequences of these changes typically required many steps, including mutagenesis of the gene encoding the protein, protein expression and purification, and cellular or in vitro assays. For example, an alanine scan is a method devised to assess the changes of each amino acid in a given protein to alanine (Cunningham and Wells 1989). Applying this approach to a protein of 100 amino acids would generate 1900 missense variants (100 amino acids $\times 19$ changes). The overall analysis would require months to carry out the mutagenesis step at each of the 100 codons and the subsequent individual assay of each variant. Clearly, determining the consequence of all possible variants is not tenable here. With the advent and combination of developing technologies, however, $>10^{5}$ variants of a protein now can be scored for function in parallel in a single experiment (Fowler et al. 2010; Hietpas et al. 2011). Because of the depth of mutational coverage and the analyses they enable, these experiments yield a wealth of knowledge, providing insight into the in vivo and in vitro properties of the proteins under study. These high-throughput approaches are particularly powerful in Saccharomyces cerevisiae, given the multitude of genetic selections available for this organism.

\section{DEEP MUTATIONAL SCANNING}

Deep mutational scanning combines a genotype-phenotype system with high-throughput DNA sequencing (Araya and Fowler 2011). Variants of a protein expressed in such a coupled system can be

\footnotetext{
${ }^{4}$ Correspondence: fields@uw.edu; Istarita@uw.edu

(c) 2015 Cold Spring Harbor Laboratory Press

Cite this introduction as Cold Spring Harb Protoc; doi:10.1101/pdb.top077503
} 
subjected to a selection dependent on the protein's function, such that variants that perform well increase in abundance and those that perform poorly decrease. These changes in frequency can be assessed by sequencing the DNA that encodes the variants, both before and after the selection step (simplified cartoon, Fig. 1A). A score, called the enrichment ratio, provides a measurement of each variant's function. The ratio for each variant is calculated by dividing the frequency of a variant in the selected population by its frequency in the starting or input population. An enrichment ratio of 1 , representing no change in a variant's abundance during the selection, indicates that a mutation is neutral; an enrichment ratio of $>1$, representing an increase in abundance, indicates that a mutation is beneficial; and an enrichment ratio of $<1$, representing a decrease in abundance, indicates that a mutation is deleterious. These scores can be visualized as a sequence-function map (Fig. 1B).

Assays that couple genotype to phenotype and are therefore amenable to this approach are diverse. For example, a protein essential for yeast growth under a specified condition can be expressed from a plasmid-encoded gene (Hietpas et al. 2011; Kim et al. 2013; Melamed et al. 2013; Roscoe et al. 2013). A library of mutated versions of this gene is generated and transformed into yeast, under a selection condition that demands the transformants express the activity of the protein. Many other assays that allow the separation of functional from nonfunctional variants can be experimentally incorporated. This partitioning can be achieved by the use, for example, of fluorescent reporters followed by fluorescence-activated cell sorting or of yeast protein display and selection of functional variants by their binding to an immobilized or fluorescent ligand (Whitehead et al. 2012).

Human proteins expressed in yeast also can be subjected to deep mutational scanning assays to reveal the functional consequences of amino acid substitutions that arise from human genetic variation. The human protein cystathionine- $\beta$-synthase (CBS), deleterious mutants of which cause homocystinuria, was used in one such study. Because human CBS and the S. cerevisiae cystathionine$\beta$-synthase (Cys4) perform the same function-synthesis of cysteine and glutathione-expression of human CBS in yeast can complement the deletion of yeast CYS4 (Kruger and Cox 1994). In a crossspecies complementation experiment, 84 alleles of human $C B S$ were tested for their ability to support the growth of yeast on media lacking glutathione (Mayfield et al. 2012). Extending this approach to a deep mutational scan of CBS would entail scoring every possible missense mutation for function, resulting in a prediction for function of mutant alleles before they are found in the human population. Even human proteins that do not have orthologous proteins in yeast may be amenable to many yeast-based technologies, including one-hybrid assays to test for transcriptional activity, twohybrid assays to detect protein-protein interaction, and three-hybrid assays to analyze proteinRNA interaction.

Carrying out a mutational scan under differing conditions can reveal various features of a protein's interaction network. The interactions between a protein of interest and other genes or pathways may

A

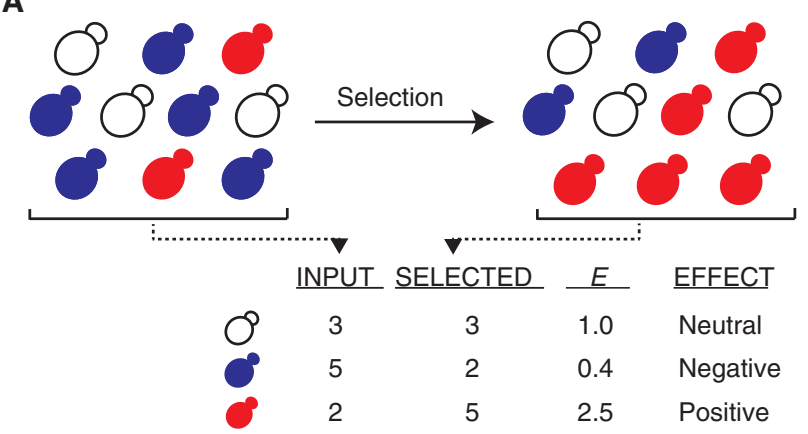

B

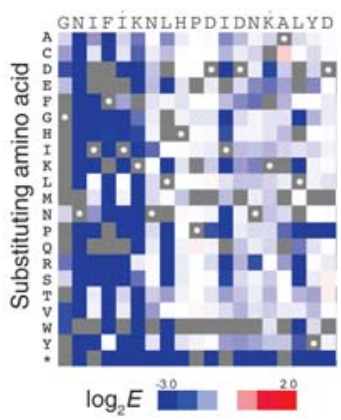

FIGURE 1. Deep mutational scanning. (A) Schematic of a deep mutational scanning experiment. Change in the abundance of variants is tracked by next-generation sequencing of DNA variants before and after selection for the function of the protein. The enrichment ratio $(E)$ is a measure of the performance of each variant. $(B)$ A sequencefunction map of $\log _{2}$ enrichment ratios $(E)$ for variants with a single amino acid change in a portion of the Pab1 protein. Blue, white, and red boxes represent variants that were depleted, neutral, or enriched, respectively, during the selection process; gray represents that no data passed quality filters; boxed with white dots represent the wild-type residue. 
be identified by performing selections in different genetic backgrounds. For example, a deep mutational scan of a protein performed both in wild-type yeast and in a strain deleted for a specific chaperone protein may reveal mutations that affect the client (partner) status of the chaperone. An increase in temperature or the addition of a denaturant during selection can exacerbate protein misfolding, and thus be used to identify mutations that affect folding (Traxlmayr et al. 2012). Chemical interactions can also be probed by the addition of small molecules to the selection. In this case, a protein's resistance profile to a specific drug may be determined by performing a deep mutational scan in the presence and absence of the drug.

Protein engineering is another field in which deep mutational scanning has provided insights. For example, in an experiment to analyze binding affinity, a protein designed to bind to the influenza virus hemagglutinin was scanned using a yeast display selection. The protein variants were analyzed to determine the positions of amino acid changes that resulted in increased binding to the viral protein (Whitehead et al. 2012). The results of this experiment were used to identify a new protein having changes at five positions with a concomitant 25 -fold increase in binding affinity.

\section{INFORMATION FOUND IN SEQUENCE-FUNCTION MAPS}

Aspects of the structure and function of a protein also can be gleaned from the sequence-function maps generated by deep mutational scanning. In the most basic analysis, regions of the protein that are critical for function will be revealed as the most intolerant to substitution. For example, the catalytically important positions in an enzyme typically are intolerant to many changes. In contrast, rare substitutions that enhance catalytic activity may be identified in a deep mutational scan. These variants can be used to probe the biochemical mechanisms of catalysis (Starita et al. 2013) and may be industrially relevant. There are other regions of a given protein-outside the catalytic core - that will tolerate only a subset of amino acid substitutions. For example, a protein may tolerate the substitution of amino acids that make up its hydrophobic core for other hydrophobic amino acids, whereas changes in these positions to charged amino acids may cause the protein to become unstable (Fowler et al. 2010; Melamed et al. 2013; Roscoe et al. 2013).

Enrichment ratios of variants containing more than one amino acid substitution can reveal mutations that show epistasis in the presence of other mutations. For example, an amino acid substitution that thermodynamically stabilizes a protein can be identified as a mutation that rescues another deleterious mutation when the two mutations are paired in a double mutant (Araya et al. 2012).

Yeast should continue to be a proving ground for new uses of deep mutational scanning approaches. The combination of the many genetic strategies and resources that are available in yeast, combined with the ingenuity of yeast researchers, are likely to ensure that this organism remains the model in deciphering protein function at high throughput. In the associated protocols, we discuss the unique challenges of performing deep mutational scanning experiments in yeast (see Protocol: Deep Mutational Scanning: Library Construction, Functional Selection, and High-Throughput Sequencing [Starita and Fields 2015a] and Protocol: Deep Mutational Scanning: Calculating Enrichment Scores for Protein Variants from DNA Sequencing Output Files [Starita and Fields 2015b]).

\section{REFERENCES}

Araya CL, Fowler DM. 2011. Deep mutational scanning: Assessing protein function on a massive scale. Trends Biotech 29: 435-442.

Araya CL, Fowler DM, Chen W, Muniez I, Kelly JW, Fields S. 2012. A fundamental protein property, thermodynamic stability, revealed solely from large-scale measurements of protein function. Proc Natl Acad Sci 109: 16858-16863.

Cunningham BC, Wells JA. 1989. High-resolution epitope mapping of hGH-receptor interactions by alanine-scanning mutagenesis. Science 244: 1081-1085.
Fowler DM, Araya CL, Fleishman SJ, Kellogg EH, Stephany JJ, Baker D, Fields S. 2010. High-resolution mapping of protein sequence-function relationships. Nat Meth 7: 741-746.

Hietpas RT, Jensen JD, Bolon DNA. 2011. Experimental illumination of a fitness landscape. Proc Natl Acad Sci 108: 7896-7901.

Kim I, Miller CR, Young DL, Fields S. 2013. High-throughput analysis of in vivo protein stability. Mol Cell Prot 12: 3370-3378.

Kruger WD, Cox DR. 1994. A yeast system for expression of human cystathionine beta-synthase: Structural and functional conser- 
L.M. Starita and S. Fields

vation of the human and yeast genes. Proc Natl Acad Sci 91: 66146618.

Mayfield JA, Davies MW, Dimster-Denk D, Pleskac N, McCarthy S, Boydston EA, Fink L, Lin AS, Meighan M, Rine J. 2012. Surrogate genetics and metabolic profiling for characterization of human disease alleles. Genetics 190: 1309-1323.

Melamed D, Young DL, Gamble CE, Miller CR, Fields S. 2013. Deep mutational scanning of an RRM domain of the Saccharomyces cerevisiae Poly(A)-binding protein. RNA 19: 1537-1551.

Roscoe BP, Thayer KM, Zeldovich KB, Fushman D, Bolon DNA. 2013. Analyses of the effects of all ubiquitin point mutants on yeast growth rate. J Mol Biol 425: 1363-1377.

Starita LM, Fields S. 2015a. Deep mutational scanning: Library construction, functional selection, and high-throughput sequencing. Cold Spring Harb Protoc doi: 10.1101/pdb.prot085225.
Starita LM, Fields S. 2015b. Deep mutational scanning: Calculating enrichment scores for protein variants from DNA sequencing output files. Cold Spring Harb Protoc doi: 10.1101/pdb.prot085233.

Starita LM, Pruneda JN, Lo RS, Fowler DM, Kim HJ, Hiatt JB, Shendure J, Brzovic PS, Fields S, Klevit RE. 2013. Activity-enhancing mutations in an E3 ubiquitin ligase identified by high-throughput mutagenesis. Proc Natl Acad Sci 110: E1263-E1272.

Traxlmayr MW, Hasenhindl C, Hackl M, Stadlmayr G, Rybka JD, Borth N, Grillari J, Rüker F, Obinger C. 2012. Construction of a stability landscape of the $\mathrm{CH} 3$ domain of human IgG1 by combining directed evolution with high throughput sequencing. J Mol Biol 423: 397-412.

Whitehead TA, Chevalier A, Song Y, Dreyfus C, Fleishman SJ, De Mattos C, Myers CA, Kamisetty H, Blair P, Wilson IA, Baker D. 2012. Optimization of affinity, specificity and function of designed influenza inhibitors using deep sequencing. Nat Biotech 30: 543-548. 


\title{
Deep Mutational Scanning: A Highly Parallel Method to Measure the Effects of Mutation on Protein Function
}

\author{
Lea M. Starita and Stanley Fields
}

Cold Spring Harb Protoc; doi: 10.1101/pdb.top077503

\begin{tabular}{|c|c|}
\hline $\begin{array}{l}\text { Email Alerting } \\
\text { Service }\end{array}$ & Receive free email alerts when new articles cite this article - click here. \\
\hline $\begin{array}{l}\text { Subject } \\
\text { Categories }\end{array}$ & $\begin{array}{l}\text { Browse articles on similar topics from Cold Spring Harbor Protocols. } \\
\text { Bioinformatics/Genomics, general (192 articles) } \\
\text { Characterization of Proteins (208 articles) } \\
\text { Computational Biology (100 articles) } \\
\text { High-Throughput Analysis, general (155 articles) } \\
\text { Mutagenesis (57 articles) } \\
\text { Proteins and Proteomics, general (575 articles) } \\
\text { Yeast (288 articles) }\end{array}$ \\
\hline
\end{tabular}

